\title{
Chirp filtering in the fractional Fourier domain
}

\author{
Rainer G. Dorsch, Adolf W. Lohmann, Yigal Bitran, David Mendlovic, \\ and Haldun M. Ozaktas
}

\begin{abstract}
In the Wigner domain of a one-dimensional function, a certain chirp term represents a rotated line delta function. On the other hand, a fractional Fourier transform (FRT) can be associated with a rotation of the Wigner-distribution function by an angle connected with the FRT order. Thus with the FRT tool a chirp and a delta function can be transformed one into the other. Taking the chirp as additive noise, the FRT is used for filtering the line delta function in the appropriate fractional Fourier domain. Experimental filtering results for a Gaussian input function, which is modulated by an additive chirp noise, are shown. Excellent agreement between experiments and computer simulations is achieved.

Key words: Filtering, Fourier optics, fractional Fourier transform.
\end{abstract}

\section{Introduction}

The fractional Fourier transform (FRT), which is a generalization of the Fourier transform, was first introduced mathematically by Namias ${ }^{1}$ and by McBride and Kerr. ${ }^{2}$ Recently, optical interpretations of the FRT were suggested and found to be equivalent to the mathematical definitions. ${ }^{3}$ Two definitions were suggested: the first is based on a graded-index medium, ${ }^{4}$ and the other is based on the Wignerdistribution function ${ }^{5}$ WDF). The WDF approach is better suited for bulk-optics implementation, and thus we concentrate on it. A short introduction of the Wigner transform and its relation to the FRT are presented. Background information about the WDF can be found in Ref. 6 .

The Wigner transform of a one-dimensional function $u(x)$ is defined by

$$
\begin{aligned}
W_{0}(x, v)= & \int u\left(x+x^{\prime} / 2\right) u^{*}\left(x-x^{\prime} / 2\right) \\
& \times \exp \left(-2 \pi i v x^{\prime}\right) \mathrm{d} x^{\prime},
\end{aligned}
$$

where $x$ represents the space (or time) coordinate and $v$ represents the frequency. The inversion from the

R. G. Dorsch and A. W. Lohmann are with the Angewandte Optik, Erlangen University, Erlangen 91058, Germany; Y. Bitran and D. Mendlovic are with the Faculty of Engineering, Tel-Aviv University, Tel-Aviv 69978, Israel; H. M. Ozaktas is with the Department of Electrical Engineering, Bilkent University, Bilkent 06533, Ankara, Turkey.

Received 16 March 1994; revised manuscript received 26 May 1994.

0003-6935/94/327599-04\$06.00/0.

(C) 1994 Optical Society of America.
WDF to the signal is unique, apart from a constant phase factor:

$$
\int W(x, v) \exp (4 \pi i x \nu) \mathrm{d} \nu=u(2 x) u^{*}(0) .
$$

The Wigner transform describes the space and the frequency contents of a signal $u(x)$, with $W\left(x_{0}, v_{0}\right)$ describing the energy of the signal $u$ at a certain point (or time) $x_{0}$ for a certain frequency $\nu_{0}$. Well-known physical properties of the Wigner distribution $W(x, v)$ include the following:

$$
\int W(x, v) \mathrm{d} x=|\tilde{u}(v)|^{2}
$$

(spectrum),

$$
\int W(x, v) \mathrm{d} v=|u(x)|^{2}
$$

(intensity),

$$
\iint W(x, v) \mathrm{d} x \mathrm{~d} v=E_{\text {total }}
$$

(energy). 6 :

Some relevant examples of WDF's are given in Ref.

$$
f(x)=\delta\left(x-x_{0}\right) \Rightarrow W_{0}(x, v)=\delta\left(x-x_{0}\right)
$$

(pulse)

$$
f(x)=\exp \left(2 \pi i \nu_{0} x\right) \Rightarrow W_{0}(x, v)=\delta\left(\nu-v_{0}\right),
$$


(monofrequency)

$$
\begin{aligned}
f(x) & =\exp \left[2 \pi i\left(b_{2} x^{2} / 2+b_{1} x+b_{0}\right)\right] \Rightarrow W_{0}(x, \nu) \\
& =\delta\left(b_{2} x+b_{1}-\nu\right) .
\end{aligned}
$$

[linear increasing frequency (chirp function)],

The above examples can be interpreted as follows: a delta function corresponds to a vertical line in the Wigner domain. A single frequency corresponds to a horizontal line, while a chirp function corresponds to a rotated line. Thus a $\pi / 2$ rotation operation in the Wigner domain transforms a pulse to a monofrequency function, passing through chirp functions. A deeper insight into the underlying theory is given in Ref. 7.

The Fourier transform $\tilde{u}(\nu)$ of $u(x)$ is given by

$$
\tilde{u}(r)=\int u(x) \exp (-2 \pi i x v) \mathrm{d} x .
$$

By replacing the input $u$ by its Fourier transform $\tilde{u}$, we can rotate the WDF by $90^{\circ}$.

$$
u(x) \rightarrow \tilde{u}(\nu): \quad W_{0}(-v, x) .
$$

The definition of the fractional Fourier transform is related to a rotation of the corresponding WDF by an angle other than $90^{\circ}$, say, $\phi=P \pi / 2$. Expressed indirectly,

$$
\mathscr{W}\left[\mathscr{F}^{P}(u)\right]=R_{-\phi} \mathscr{W}[u],
$$

where $R_{\phi}$ denotes a counterclockwise rotation of a two-dimensional function and $\mathscr{W}[u]$ is the Wigner transform of $u(x)$. This definition satisfies three basic requirements. ${ }^{3-5}$

(1) The FRT is linear in $u(x)$.

(2) The FRT of order $P=1$ is the Fourier transform.

(3) Repeated applications of the FRT are additive in $P: \quad \mathscr{F}^{P_{1}} \mathscr{F} P_{2} u(x)=\mathscr{F}^{P_{1}+P_{2}} u(x)$.

Rotation of the WDF can be expressed as three shearing operations $(x, v$, and $x$ shearings, or $v, x$, and $\nu$ shearings). ${ }^{5}$ Thus it is suggested in Ref. 5 that the system of Fig. 1 be used to implement the fractional Fourier transform by optical means. The $x$ shearing is performed by free-space propagation, the lens then performs $v$ shearing, and $x$ shearing is again per-

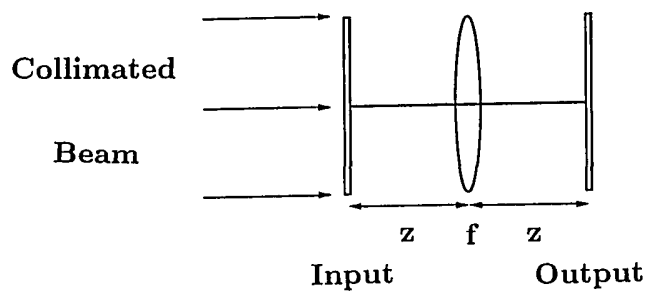

Fig. 1. Optical setup for performing a FRT.

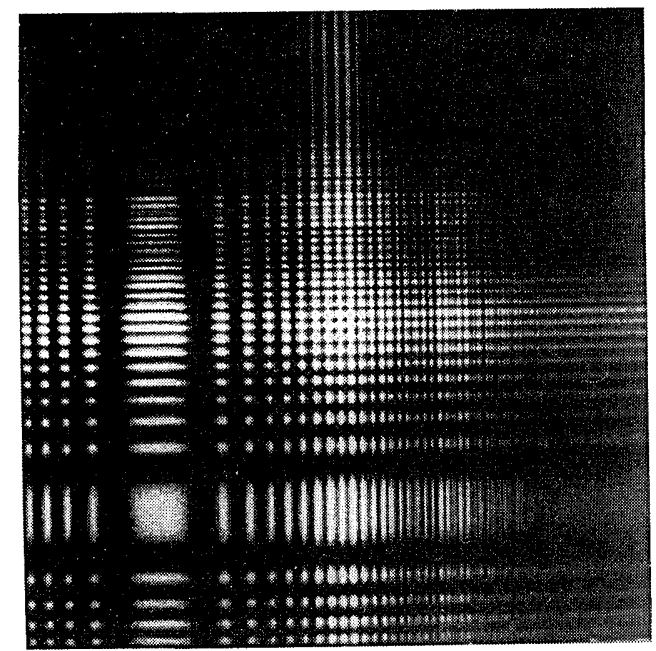

Fig. 2. Computer simulation of the input pattern.

formed by free-space propagation. The effect of propagation of a signal $u_{0}(x)$ through this system is described mathematically by

$$
\begin{aligned}
\mathscr{F}^{P}\left[u_{0}(x)\right]= & u_{P}(x)=\int_{-\infty}^{\infty} u_{0}\left(x_{0}\right) \exp \left[i \pi\left(\frac{x^{2}+x_{0}^{2}}{T}\right)\right] \\
& \times \exp \left(-i 2 \pi \frac{x_{0}}{S}\right) \mathrm{d} x_{0},
\end{aligned}
$$

with

$$
T=\lambda f_{1} \tan (\phi), \quad S=\lambda f_{1} \sin (\phi),
$$

where $\lambda$ is the wavelength of light and $f=f_{1} / \tan (\phi / 2)$. The free-space propagation distance $z$ is given by

$$
z=f_{1} \sin (\phi) .
$$

Section 2 describes an interesting application of the

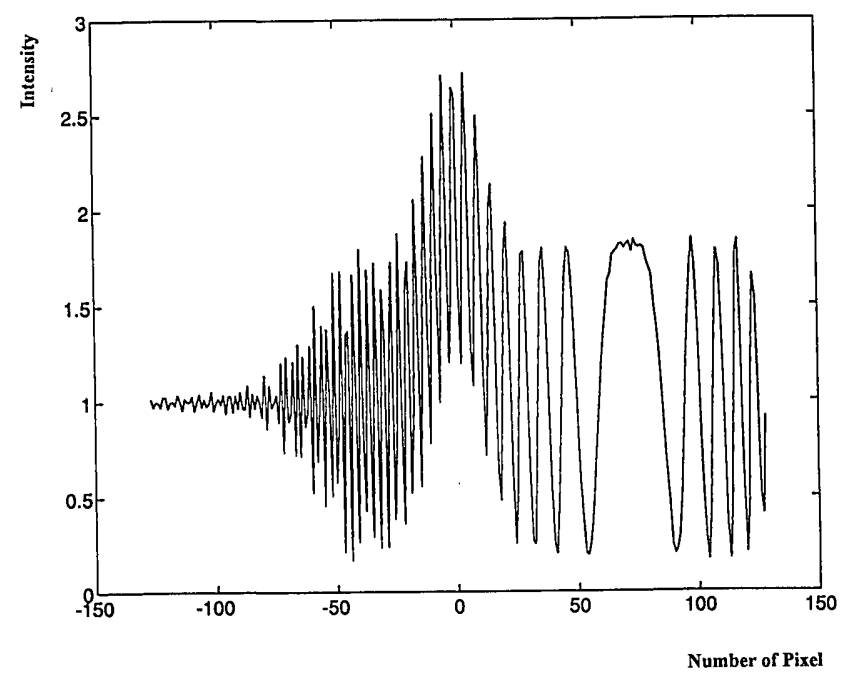

Fig. 3. Computer simulation of the input pattern, shown by a cross section through the center [the chirp is added to the Gaussian plus a constant, according to Eq. (16)]. 


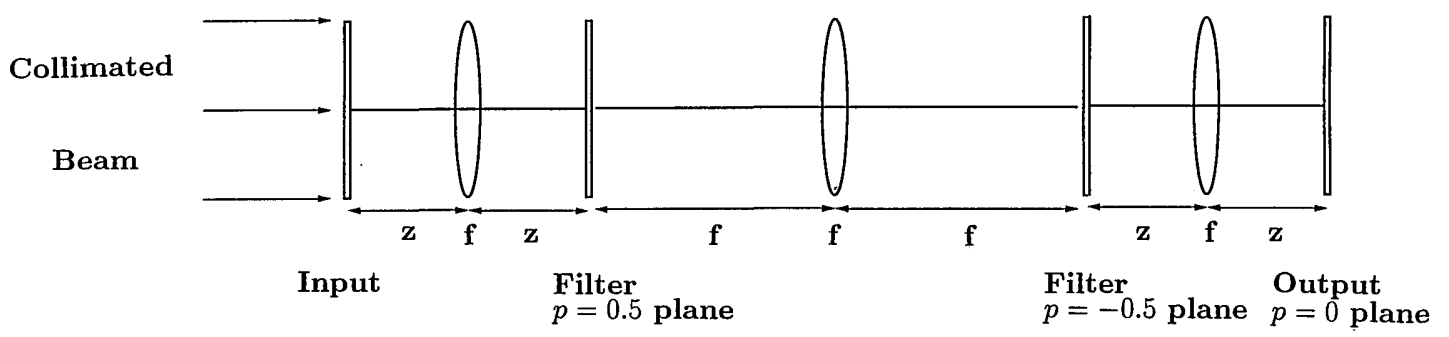

Fig. 4. Optical setup for performing the chirp filtering.

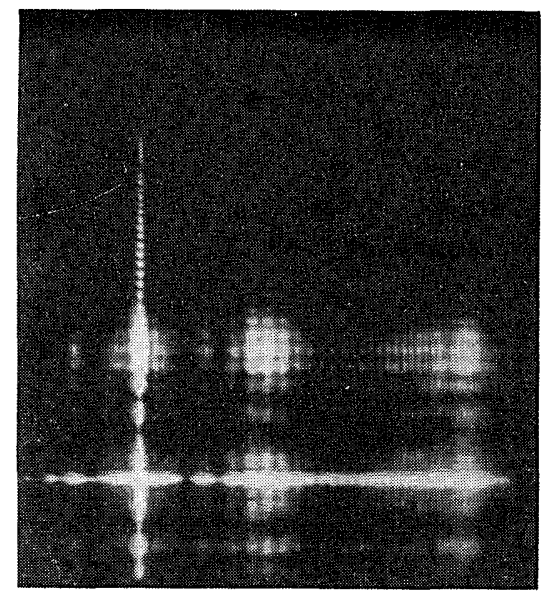

Fig. 5. Experimental intensity distribution of the $P=+0.5$ plane.

FRT. We address the question how to filter such a chirp function when regarded as additive noise.

\section{Filtering Additive Chirp Noise}

Equation (8) and relation (11) imply that, by performing an FRT of a certain order $P$, we can transform a chirp term into a delta function. The fractional order $P$ is determined by ${ }^{7}$

$$
\phi=\tan ^{-1} b_{2},
$$

while $P=\phi /(\pi / 2)$.

To demonstrate the application of the FRT to chirp filtering, we choose the following real function as the input function:

$$
u_{0}(x)=\exp \left(-\pi x^{2}\right)+\cos \left[2 \pi\left(\frac{x^{2}}{2}-4 x\right)\right]+C .
$$

The signal consists of a Gaussian term, a constant term $C$, and an unwanted noise term (in brackets). The cosine function can be broken into two complex exponential chirps:

$$
\begin{aligned}
\cos \left[2 \pi\left(\frac{x^{2}}{2}-4 x\right)\right]= & \frac{1}{2} \exp \left[i 2 \pi\left(\frac{x^{2}}{2}-4 x\right)\right] \\
& +\frac{1}{2} \exp \left[-i 2 \pi\left(\frac{x^{2}}{2}-4 x\right)\right] .
\end{aligned}
$$

The first chirp corresponds to a delta function in

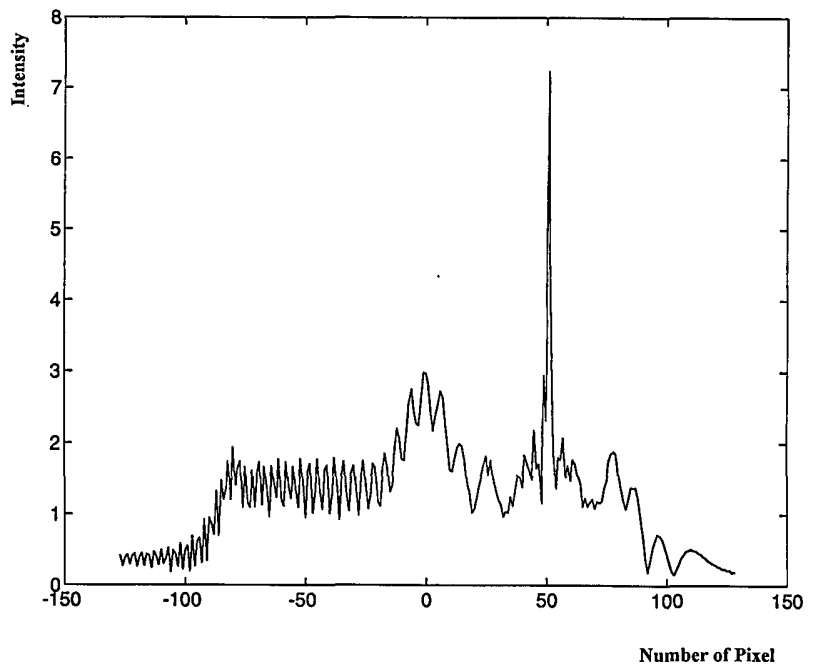

Fig. 6. Computer simulation of Fig. 5 (cross section), showing the chirp turned into a delta peak by means of an FRT.

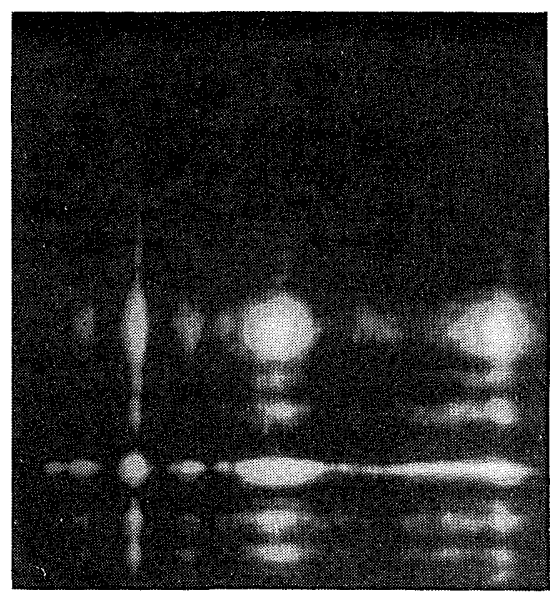

Fig. 7. Experimental intensity distribution of the $P=-0.5$ plane.

the $P=+0.5$ domain, and the other corresponds to a delta function in the $P=-0.5$ domain. Therefore the filtering of the chirp is done by application of a band-stop mask in each of these fractional domains. The processing algorithm consists of the following steps:

(1) Perform an FRT of order $P=0.5$.

(2) Multiply by the band-stop filter.

(3) Inverse Fourier transform to the $P=-0.5$ domain. 


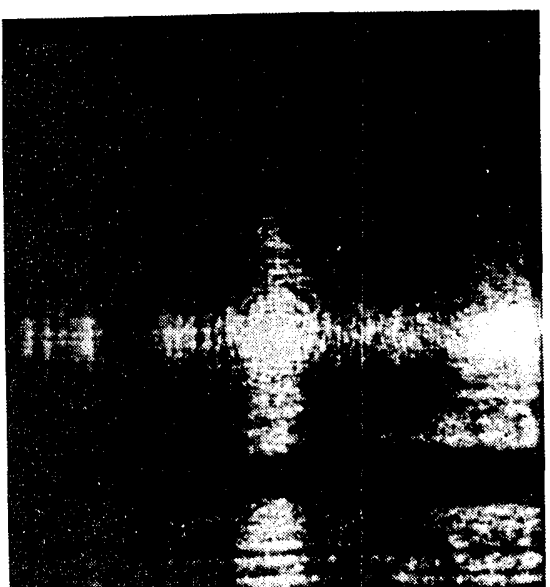

Fig. 8. Experimental intensity distribution of the output plane.

(4) Multiply by the band-stop filter.

(5) Perform an FRT of order 0.5 to the $P=0$ domain.

In our experiment we generated a line delta function and performed an FRT of degree $P=0.5$. The resulting chirp is added to a Gaussian function with a dc. The resulting pattern was used as input for our filtering experiment and is shown in Fig. 2. A cross section is displayed in Fig. 3.

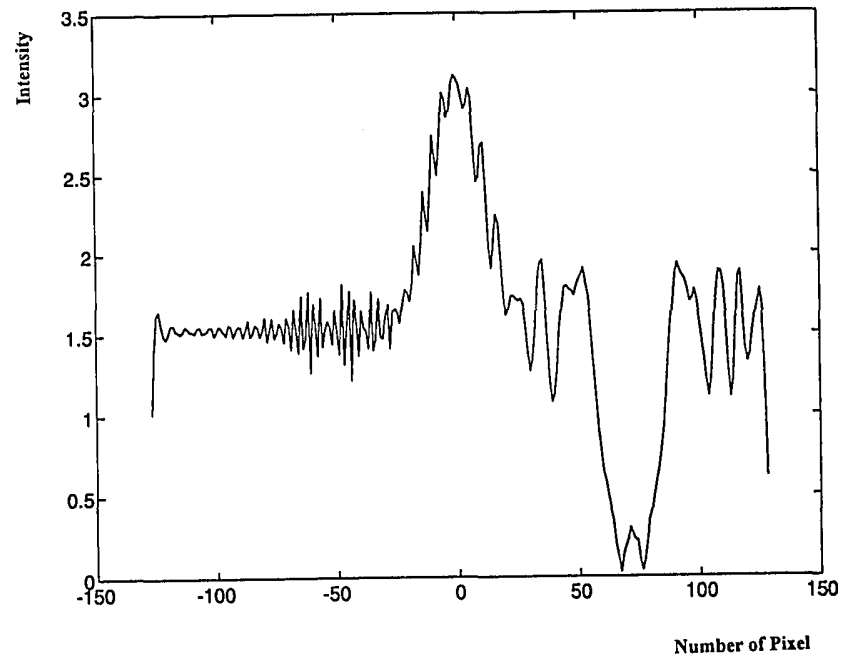

Fig. 9. Computer simulation of Fig. 8 (cross section), showing the filtered Gaussian function at the output plane.
The optical setup to perform the total filtering is shown in Fig. 4. The first step is a 0.5 FRT according to Fig. 1. The experimental intensity distribution in this plane is shown in Fig. 5. A cross section of the corresponding computer simulation is displayed in Fig. 6. The band-stop mask placed in the +0.5 plane is a simple binary off-center cross. The experimental intensity distribution of the -0.5 plane is shown in Fig. 7. Here the second mask is used. An additional fractional Fourier transform of degree $P=0.5$ leads to an overall transform of $P=2$ or, equivalently, by $P=0$. The experimental intensity distribution of the final output plane is shown in Fig. 8. The chirp is filtered out perfectly. The desired chirp-free Gaussian distribution remains. Our computer simulation of the final output, displayed as a cross section in Fig. 9, indicates a very good agreement with the experimental results.

\section{Conclusion}

In this paper we have presented an application of the fractional Fourier transform (FRT) in optical onedimensional signal processing. The FRT is used to perform a transform from a chirp function to a line delta function. A spatial filter process is applyed to eliminate the chirp when treated as noise. Experimental realization for a specific function was demonstrated.

\section{References}

1. V. Namias, "The fractional order Fourier transform and its application to quantum mechanics," J. Inst. Math. Appl. 25, 241-265 (1980).

2. A. C. McBride and F. H. Kerr, "On Namias's fractional Fourier transforms," IMA J. Appl. Math. 39, 159-175 (1987).

3. D. Mendlovic, H. M. Ozaktas, and A. W. Lohmann, "Gradedindex media, Wigner-distribution functions, and the fractional Fourier transform," Appl. Opt. 33, 6182-6187.

4. D. Mendlovic and H. M. Ozaktas, "Fractional Fourier transformations and their optical implementation. Part I," J. Opt. Soc. Am. A 10, 1875-1881 (1993).

5. A. W. Lohmann, "Image rotation, Wigner rotation, and the fractional Fourier transform," J. Opt. Soc. Am. A 10, 21812186 (1993).

6. H. O. Bartelt, K.-H. Brenner, and A. W. Lohmann, "The Wigner distribution function and its optical production," Opt. Commun. 32, 32-38 (1980).

7. H. M. Ozaktas, B. Barshan, D. Mendlovic, and L. Onural, "Convolution, filtering, and multiplexing in fractional Fourier domain and their relation to chirp and wavelet transform," J. Opt. Soc. Am. A 11, 547-559 (1994). 